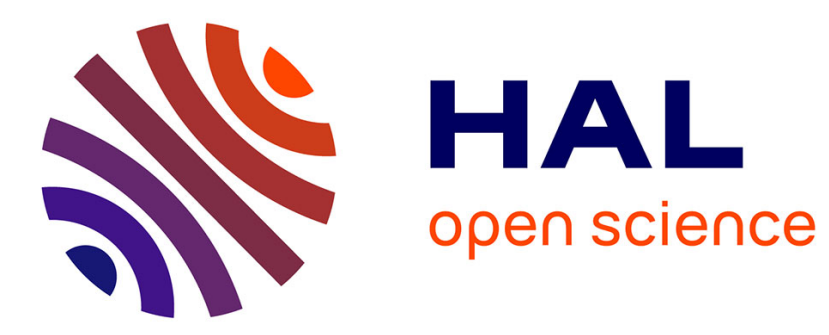

\title{
Rigorous Simulation of Line-Defects in Extreme UV Masks
}

Patrick Schiavone, Renaud Payerne

\section{To cite this version:}

Patrick Schiavone, Renaud Payerne. Rigorous Simulation of Line-Defects in Extreme UV Masks. Japanese Journal of Applied Physics, 2005, Vol. 44 (9A), pp.6810-6816. 10.1143/JJAP.44.6810 . hal-00020640

\section{HAL Id: hal-00020640 \\ https://hal.science/hal-00020640}

Submitted on 13 Mar 2006

HAL is a multi-disciplinary open access archive for the deposit and dissemination of scientific research documents, whether they are published or not. The documents may come from teaching and research institutions in France or abroad, or from public or private research centers.
L'archive ouverte pluridisciplinaire HAL, est destinée au dépôt et à la diffusion de documents scientifiques de niveau recherche, publiés ou non, émanant des établissements d'enseignement et de recherche français ou étrangers, des laboratoires publics ou privés. 


\title{
Rigorous simulation of line-defects in EUV masks
}

\author{
Patrick Schiavone, Renaud Payerne \\ Laboratoire des Technologies de la Microélectronique CNRS, c/o CEA-Grenoble, 17 rue des \\ Martyrs, 38054 GRENOBLE Cedex 9, France
}

\begin{abstract}
:
In this paper, rigorous electromagnetic simulation is used to investigate the behavior of line defects in EUV masks. Using the Modal Method by Fourier Expansion, the geometry of the structure as well as the polarization state can be handled. A simple analytical model has been developed in order to generate the input geometry for the simulations. The deposition conditions can be mimicked by changing an empirical parameter representing the planarisation properties of the process. The influence of the defect size and of its position in the multilayer mirror is analyzed. The position of the defect with respect to an absorber feature is very important and is also considered.

It is shown that the size of the nucleation particle by itself is not a relevant parameter to describe a defect. The process conditions modify largely the propagation of the defect into the multilayer mirror and induce very large variation of its printability. Some defects that do not affect the surface of the mirror can induce a non negligible intensity drop
\end{abstract}

Keywords: $\quad$ EUV lithography, mask, multilayer, defect, simulation, rigorous electromagnetic model 


\section{Introduction}

Extreme UV lithography is seen as the main candidate for the fabrication of the sub 40nm generation integrated circuits. In addition to the difficult issues to be addressed like source and reflective optics, more practical problems are to be investigated. The impact of the defects in the EUV masks is one of these critical issues. It is important to be able to define what kind of defect will be printable and inspectable. Simulation is a very powerful tool to investigate the different possible situations for the size and position of a defect (absorber, mirror, substrate defect ...) and their impact on the wafer image. The speed of our rigorous simulation allows us to make an evaluation of the problem of the defects without making too restrictive hypothesis (for example on the number of layers or on the layer geometry).

The problem of defects in EUV mask has already been addressed by several authors ${ }^{1-4)}$. In simplified approaches, the defect is only seen as a perturbation of the surface of the multilayer mirror $^{2,3)}$. It is considered as a phase shift on the reflected electric field. Rigorous computation based on finite difference schemes have the drawback of being very memory and time consuming. The use of such method in the case of EUV wavelength is thus very painful and do not easily allow parametric analysis. In this paper, we show that Modal Method by Fourier Expansion can be extended to handle the complex geometry of EUV defective masks. In the first part, we describe a very simple analytical model that has been used to generate the input geometry for the optical simulation. It is not intended to be physically meaningful but rather to give realistic profiles of the multilayer growth over a non planar topography. An empirical parameter of this model allows representing conformal as well as planarizing deposition conditions. In the second part of the paper, the influence of the size and position of the defect in the multilayer mirror is examined. It is confirmed that the nucleating seed size and deposition strongly affects the near field at the mask and consequently the aerial image at the wafer. The deposition conditions have also a significant influence on the impact of a given defect. As already shown by Bollepali et al. ${ }^{5)}$, in certain 
conditions, a multilayer deformation that does not even affect the surface of the mirror are shown to be able to cause non negligible intensity drops.

\section{Multilayer deposition simplified model:}

In order to generate easy to use input topographies for the optical calculation, we have developed a simple model of multilayer deposition. It is not intended to give deep physical insight of the deposition process, but rather to give an empirical description of the geometry of the multilayer deposited on top of a defect seed.

This model is based on the classical problem of parallel curves ${ }^{6}$. The idea first came by considering that in the case of perfectly conformal deposition above a shape with smooth enough shape, the resulting top surface of the layer is strictly parallel to the bottom shape. This problem has been addressed a few centuries ago by Leibniz in the case of a curve described by its parametric equations. In this paper, we always deal with circles or ellipses but other shapes can be handled as well.

The parametric equation of an ellipse placed just above the axis $y=0$ is given by equation (1) where $a$ is the half length of the ellipse in the $\mathrm{x}$ direction and $b$ is the half length of the ellipse in the $y$ direction. The $x$ and $y$ directions are respectively parallel and perpendicular to the multilayer surface. In the following, all objects are considered to be invariant along the $z$ direction.

$\left\{\begin{array}{c}x(t)=a \cos (t) \\ y(t)=b \sin (t)+b\end{array}\right.$

the parametric equation of the curve parallel to the curve defined by $x(t)$ and $y(t)$ sitting at a distance $d$ from it can be written:

$$
\left\{\begin{array}{l}
x p(t)=x(t)+d \frac{y^{\prime}(t)}{\sqrt{x^{\prime}(t)^{2}+y^{\prime}(t)^{2}}} \\
y p(t)=y(t)-d \frac{x^{\prime}(t)}{\sqrt{x^{\prime}(t)^{2}+y^{\prime}(t)^{2}}}
\end{array}\right.
$$

where $x^{\prime}(t)$ and $y^{\prime}(t)$ are respectively the derivatives of $x(t)$ and $y(t)$ with respect to the parameter $t$. 
In order to be able to represent more closely experimentally observed planarizing deposition processes $^{7)}$, equation 2 can be modified slightly by introducing the empirical parameter $\alpha$. Its practical impact is to pull up or down the "parallel" curve in the $y$ direction with respect to the $x$ direction.

$$
\left\{\begin{array}{c}
x p(t)=x(t)+d \frac{y^{\prime}(t)}{\sqrt{x^{\prime}(t)^{2}+y^{\prime}(t)^{2}}} \\
y p(t)=y(t)-\alpha \cdot d \frac{x^{\prime}(t)}{\sqrt{x^{\prime}(t)^{2}+y^{\prime}(t)^{2}}}
\end{array}\right.
$$

Figure 1 shows the impact of $\alpha$ on the resulting curve. It can clearly be seen that tuning $\alpha$ allows to get a shape that can be thought as representing conformal $(\alpha=1)$ or planarizing $(\alpha>1)$ deposition conditions.

Equation 3 has been used to generate the input geometries that are needed for the optical simulation in this paper. In the following, we have generally taken two different values of the parameter $\alpha=1$ and $\alpha=1.05$. The former case is the situation usually encountered with a standard off-normal sputtering process (for example using Ion Beam Sputtering) where the deposited material is close to conformal. The latter occurs when using for example near normal incidence sputtering in association with milling steps that has the capability of gradually flattening the multilayer surface ${ }^{11)}$, using $\alpha=1.05$, a $20 \mathrm{~nm}$ circular seed is close to be completely smoothed. Figure 2 shows the full geometry that is resulting from these two types of geometries (for sake of clarity of the pictures, not all layers have been drawn). The size of the initial circular defect is $20 \mathrm{~nm}$. It can be seen that the top surface height variation is equal to the defect size in the conformal case whereas no surface bump is produced in the planarizing deposition condition (here $\alpha=1.07$ ). The height and width of the deformation induced at the top surface of a 40 layer pair mirror for different planarization coefficients is shown in Figure 7. The defect size at the substrate interface ranges between 20 and $100 \mathrm{~nm}$. 


\section{Simulation method:}

\subsection{The Modal Method by Fourier Expansion (MMFE):}

The basic principle of the MMFE has been already summarized in a previous paper ${ }^{8)}$ and will not be detailed here. The main characteristic of this computation is that the whole structure (considered as periodic in the $\mathrm{x}$ direction) is cut in slices $^{9)}$. Each slice is considered as a lamellar grating comprising two or more refractive indices. Maxwell equations are solved in each slice in order to get the eigenfunctions and eigenvalues. Scattering matrix formalism is used to deal with the boundary conditions at each layer interface. The diffraction efficiencies of the mask feature as well as the electromagnetic field within the structure can be computed.

An example of the slicing is shown in Figure 3 where the structure has been cut into 3 slices. In the case of a EUV multilayer defective geometry it happens that one lamellar grating can be composed of more than two different refractive indices, this can be handled without problem by the MMFE. Convergence checks have shown that 3 to 4 slices per physical layer of the mask $(\sim \lambda / 10$ slice thickness) are sufficient to get a good description of the structure by the stair step approximation. It means that for a typical EUV mask with 40 layer pairs, the number of slices to consider is in the range of 300 .

The electromagnetic field within and just above the mask (called near field) is computed. The number of retained order is $2 \mathrm{M}+1$, all the results presented in this paper use $\mathrm{M}=25$. This choice is dictated by keeping a good compromise between accuracy and speed. It has been checked that the aerial image remains unchanged if the number of orders is increased, even if slight differences appear in the near field.

\subsection{Aerial image:}

The electromagnetic field at the mask is very difficult to be measured, thus cannot be used for experimental comparison purpose. The so-called aerial image, i.e. the light intensity distribution 
computed at the wafer (image of the mask through the stepper lens) quantifies the light that exposes the photoresist. It is able to provide reliable first order information on the developed resist image. In order to compute the aerial image, the near field computed as described in the previous section is used as an input for a commercial lithography simulator. The electromagnetic field at the mask surface is considered as the complex transfer function of an equivalent phase shift mask. A proper format conversion is used so that we get an equivalent transmission mask that is handled by the commercial software in a usual way. The aerial image is computed using the Hopkins approach. This is not the most accurate way of doing, but as shown by Otaki ${ }^{10)}$, this provides results that are close from the one obtained using the rigorously computed diffraction pattern. The EUV optical system is considered to provide a $4 \mathrm{x}$ demagnification. A simple threshold model is used for the resist development. We used a threshold of 0.25 .

\section{Results:}

Our simulation code based on MMFE is fast enough (within one minute for the simulation of the reflected field of a 40 deformed layer pair blank underneath a patterned absorber using a Pentium III $800 \mathrm{MHz}$ computer). This allows an easy investigation of the different parameters of the mask geometry ${ }^{8)}$. In this paper, we investigate the influence of the defect size and position in the multilayer mirror as well as the effect of the deposition conditions.

It has to be noticed that the model used here is only two dimensional; this means that every feature is a cylinder with its axis along the $\mathrm{z}$ direction. Therefore these results do not allow drawing quantitative information about practical and useful quantities such as the minimal printable defect size because real defects are point defects, not line defects. Approximate quantitative evaluation about the $3 \mathrm{D}$ behavior can nevertheless be drawn. Indeed, as pointed out by Pistor ${ }^{1)}$ it can be considered that a point defect will cause an intensity drop that is as a rule of thumb twice smaller than that caused by a line defect of the same size. This is particularly true for mid-size objects. Anyway, these $2 \mathrm{D}$ results give very useful insight about the way defect size or deposition process 
qualitatively influence the electromagnetic near field at the mask as well as the aerial image at the wafer.

Otherwise specified, the following parameters have been used for the simulation: wavelength $13.5 \mathrm{~nm}$, incidence angle $5^{\circ}$, the mirror is made of 40 pairs of Mo $(2.85 \mathrm{~nm}) / \mathrm{Si}(4.15 \mathrm{~nm})$, the defect seed material is silicon. For aerial image computation, the numerical aperture is 0.3 and the partial coherence is 0.6 . The reduction ratio of the imaging lens is supposed to be $4 \mathrm{x}$. A perfect lens without aberration or flare is considered.

In the following, the term defect size refers to the size of the initial seed within the multilayer. To avoid any misunderstanding, the size of the perturbation at the top surface will be explicitly mentioned every time it will be used.

\subsection{Position of the defect in the multilayer mirror:}

Although it seems that most of the defects lie on the substrate, some of them can appear during the multilayer deposition process. We investigate in this section the influence of the defect within the multilayer. The aerial image are plotted in Figure 4 and Figure 5 for different planarization coefficients ( $\alpha=1$ and $\alpha=1.05$ respectively). They correspond to a $20 \mathrm{~nm}$ seed placed in different layers (from 10 to 20 pairs from the multilayer bottom. The first observation is that in both cases, the defect causes a larger intensity drop when it sits closer to the substrate. This is illustrated in the plot of the printed CD in Figure 6. The printed CD decreases when the defect sits further from the substrate.

This can appear in contradiction with an intuitive thought that considers that the defect should affect more effectively the light reflection when it sits in the upper layers of the mirror where the electromagnetic field is stronger. In fact, the above mentioned intuitive way of thinking is not true. In the configuration we are dealing with, the defect seed is small $(20 \mathrm{~nm})$ and its material is silicon which is relatively transparent in the EUV range. The cause of the intensity drop lies in the perturbed geometry of the Bragg mirror layers rather than in absorption, like it would have been the 
case in the deep UV wavelengths. In these conditions, the defect causes a more significant perturbation of the mirror topography if it lies in the lower layer of the multilayer. And consequently has a larger effect on the reflected intensity that leads to a larger printed feature. We have verified that a $20 \mathrm{~nm}$ silicon defect on top of the mirror does not induce any print on the wafer. It is too small and too transparent to have a significant effect on the reflected intensity. We have also checked that larger defect seeds (50nm and 100nm) behave similarly.

\subsection{Influence of the defect size:}

The diameter and height of the deformation of the top surface induced by defects of different size for various planarization coefficients is shown in Figure 7. For example, a 20nm seed will cause no deformation of the surface for a planarization coefficient of 1.08. For $\alpha=1$, the deformation is $105 \mathrm{~nm}$ and its height is equal to its size $20 \mathrm{~nm}$. This parameter $\alpha$ will allow us to generate geometries that affect the shape of the multilayer mirror while keeping a flat top surface.

The way the defect size affects the aerial image is shown in Figure 8 and Figure 9 for $\alpha=1$ and $\alpha=1.05$ respectively; the defect size ranges from 10 to $100 \mathrm{~nm}$. As an example, the corresponding reflected near field (that is used to generate the equivalent transmission mask) is presented in Figure 13 for $\alpha=1$ and a 40nm seed. The high frequency oscillations visible on the graph are mostly due to a numerical effect caused by the truncation of the Floquet-Fourier series. This induces a so called Gibbs effect. It can be noticed that these oscillations are not seen in the corresponding aerial images. They are filtered out due to the limited numerical aperture ( 0.3 in this case) of the EUV stepper optics.

Not surprisingly, the printed CD plotted in Figure 10 is roughly proportional to the defect size when $\alpha=1$ and line defect smaller than $20 \mathrm{~nm}$ do not cause a sufficient intensity drop for $\alpha=1.05$. The intensity drop is mainly caused by diffraction on the tilted layers of the distorted multilayer above 
the edge of the defect. This assumption is confirmed by the fact that for $\alpha=1$, the intensity curve shows a bump above the middle of the defect. At this position, the layer topography is rather flat, especially for the larger defects, less diffraction occurs.

\subsection{Defect in the vicinity of an absorber pattern}

The case of a defect in an open area discussed in the previous sections is only a part of the problem. The effect of the defect in the vicinity of an absorber pattern and the way it affects the printed CD is also very important to look at. In Figure 11 and Figure 12 the aerial images of a 20nm defect close to a 200nm chrome line (50nm @ wafer level) are plotted for two planarization coefficients $\alpha=1$ and $\alpha=1.05$. In each plot, several aerial images are drawn corresponding to varying defect positions raging from $-50 \mathrm{~nm}$ to $+100 \mathrm{~nm}$ from the line center. We can see on these graphs that when the defect lies either underneath or far $(>75 \mathrm{~nm})$ from the absorber line, the aerial image of the line is not affected. However, a significant widening is observed when the defect lies close from the absorber edge. For $\alpha=1$, the line widening is $22 \mathrm{~nm}$ and $46 \mathrm{~nm}$ when the defect is placed at a distance of $25 \mathrm{~nm}$ and $50 \mathrm{~nm}$ respectively. These line width changes reduce to $6 \mathrm{~nm}$ and $30 \mathrm{~nm}$ for a more planarizing deposition process $(\alpha=1.05)$. These large values have to be considered with care since we deal in this paper with line defect only. They are not expected to provide results that are quantitatively representative of real defects that are essentially three-dimensional.

\subsection{Printability of defects with a flat top surface}

In this paragraph, we would like to draw the attention on a result already pointed out by Bollepalli ${ }^{5)}$ concerning planarized defects which perturbations do not reach the top of the mirror. Let us take the case of an hypothetical, but nevertheless potentially existing, multilayer deposition process ${ }^{11)}$ which is such that on top of a defect, the successive layers have planarized the relief due to the defect and the top of the surface of the mirror is flat (see for example Figure $2 b$ ). The reflected near field intensity of such a defective mirror is shown in Figure 14. It can be seen that although the 
surface is flat, the reflected light suffers a large intensity drop above the defect seed. This intensity dip can surely make such a defect printable. The fact that this example is a line defect does not change the validity of this observation; a similar example with a slightly larger 3D seed would have lead to the same effect. From this fact, the question of the inspection strategy based on deep UV microscopy, as it is presently performed arises. From these simulation, as well as from some experimental work ${ }^{12)}$, it seems that an inspection tool based on actinic inspection is probably needed to catch some defect of the kind described in this section that do not perturb the surface but the underlying layers of the mirror.

\subsection{Cause of the intensity drop at the defect:}

From these simulations, it comes that two main mechanisms are the cause of the intensity drop. First, diffraction at the sloped layer interface causes a first kind of intensity loss. Second, under planarizing deposition conditions, the multilayer period on top of the defect seed is necessarily smaller than the period of the ideally matched mirror (because the total multilayer thickness is reduced). In these conditions, the mirror is not locally resonating anymore and a severe reflectivity loss is observed. This is illustrated in the two different situations which corresponding near fields (intensity and phase) are shown in Figure 13 and Figure 14. A 40nm circular seed is considered, covered by a 40 layer pair mirror. In the first case (Figure 13), $\alpha=1$, the mirror is conformally deposited, in the second one (Figure 14), $\alpha=1.18$, the planarization parameter has been chosen such that the surface of the mirror does not show any bump. In Figure 13, the intensity drop is observed at the two sides of the defect where the layer interfaces are sloped. The influence range of the defect is large, the central part of it is almost unperturbed as far as the multilayer period is considered, and moreover the layer interfaces are almost flat. This leads to an intensity that recovers almost the value of the reflectivity in the unperturbed central region. The phase, which is here unwrapped and corrected from the skew due to the oblique incidence, varies according to the surface topography. The configuration of Figure 14 is clearly different. In this case, the mirror surface is flat. The phase 
of the reflected near field varies in a small range of $40^{\circ}$. However a severe intensity drop is due to the period reduction that comes with the planarisation. Indeed in order to get a planar surface with a 20nm seed, a planarization coefficient of 1.07 or larger is required (cf. Figure 7), this mean a $7 \%$ period mismatch. Considering the high selectivity of the Bragg mirror, this is largely sufficient to cause the reflectivity to drop to a value smaller than $10 \%$.

These considerations lead to some comments about a defect mitigation strategy. It seems clear that in order to mitigate the printability of the defects, a technique that only consist in flattening the mirror surface is not foreseen to be efficient if this flattening is obtained by a process that in the same time changes the multilayer period or/and the mirror reflectivity (for example local heating of the mask). In the same way the question of the inspection strategy can be raised. Some buried defect can cause an intensity drop at the exposure wavelength without any perturbation of the mask surface. This type of defect cannot be detected using visible or DUV inspection tools and probably require inspection at the actinic wavelength. Apart from this paper, such kind of features has already been pointed out using simulation ${ }^{5)}$ and experimentally ${ }^{12)}$.

\section{Conclusion:}

The influence of line defects in a EUV multilayer has been investigated. Although the quantitative values are only valid for line defects, this study allows drawing qualitative conclusions that are also applicable to point defect. It appears that, especially for conformal deposition, defects that sit closer to the substrate lead to larger intensity drop because they induce more important topography perturbation in the multilayer mirror. Defects that are in the close vicinity of an absorber line have the larger impact on the printed CD. If the defect lies just underneath or far from the absorber pattern, it will have less impact because, either its effect on the reflected intensity is masked by the absorber when both are at the same place or, when the defect is too far, its intensity dip and the one of the absorber feature behave independently. We have also shown that the main causes of the intensity drop above a defect are twofold. Diffraction at the tilted layer interfaces is a first effect. 
Secondly, when dealing with smoothed defects, the period mismatch is the major contributor to the reflectivity loss. The defect material does not play a significant role.

Planarizing deposition conditions help in keeping a lower sensitivity to substrate defects but a flat topography does not guarantee a defect free printing. This can be considered as a serious issue and raises the question of the mask inspection. Indeed, visible or even DUV inspections are only sensitive to the blank top surface topography. At wavelength inspection ${ }^{13)}$ could be necessary to track all kind of defects in the EUV masks. Moreover, following the trend observed in the DUV lithography, at wavelength aerial image microscope is likely to be used in addition to faster inspection tools. 


\section{Figure Caption}

Figure 1: Influence of the planarisation parameter: (a) $\alpha=1$; (b) $\alpha>1$; (c) $\alpha<1$. The original shape is a circle

Figure 2: Geometry of the multilayer mirror after deposition on top of a 20nm diameter seed. (a) $\alpha=1$ (b) $\alpha=1.07$. One interface every five is shown.

Figure 3: Sketch of the structure discretisation needed for the MMFE. (a) a circular defect of refractive index $n_{2}$ is shown, 3 layers are used. (b) the corresponding simulated structure.

Figure 4: Aerial images of defective multilayers $(\alpha=1)$ as a function of the defect position within the multilayer. From the lowermost curve of the figure to the uppermost, the $20 \mathrm{~nm}$ diameter defect seed is placed respectively at $0,2,4,6,8,13,12,14,16,18,20$ layer pairs from the substrate.

Figure 5: Aerial images of defective multilayers $(\alpha=1.05)$ as a function of the defect position within the multilayer. From the lowermost curve of the figure to the uppermost, the $20 \mathrm{~nm}$ diameter defect seed is placed respectively at $0,2,4,6,8,13,12,14,16,18,20$ layer pairs from the substrate.

Figure 6:_Size of the printed feature versus seed vertical position from the substrate. $\alpha=1$ (solid line); $\alpha=1.05$ (dashed line).

Figure 7: Height (a) and diameter (b) of the deformation at the top of the mirror versus $\alpha$ for different seed sizes (from bottom to top: $20,30,40,50,60,70,80,90,100 \mathrm{~nm}$ )

Figure 8: Aerial images of defective multilayers $(\alpha=1)$. The defect seed diameters are (from top to bottom) 10, 20, 30, 50, 70, 100nm.

Figure 9: Aerial images of defective multilayers $(\alpha=1.05)$. The defect seed diameters are (from top to bottom) $10,20,30,50,70,100 \mathrm{~nm}$.

Figure 10: Size of the printed feature versus seed size. $\alpha=1$ (solid line); $\alpha=1.05$ (dashed line).

Figure 11: Aerial images of defective multilayers $(\alpha=1)$ for different relative position of the defect and the absorber; defect seed=20nm, 50nm absorber (@ wafer scale). Position of defect center (from 
the leftmost to the rightmost curve):-50,-25, 0, 25, 50, 75, 100nm (@ wafer scale) from absorber center.

Figure 12: Aerial images of defective multilayers $(\alpha=1.05)$ for different relative position of the defect and the absorber; defect seed=20nm, 50nm absorber (@wafer scale). Position of defect center (from the leftmost to the rightmost curve): -50,-25, 0, 25, 50, 75, 100nm (@wafer scale) from absorber center.

Figure 13: Reflected intensity (a) and phase (b) from a 40nm defect, $\alpha=1$.

Figure 14: Reflected intensity (a) and phase (b) from a 40nm defect, $\alpha=1.18$. 


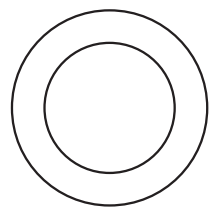

(a)

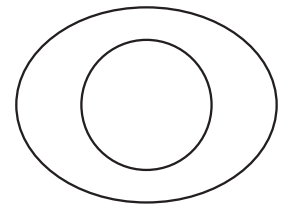

(b)

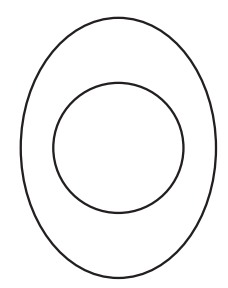

(c)

Figure 1

Patrick Schiavone, Renaud Payerne; "Rigorous simulation of line-defects in EUV masks" 


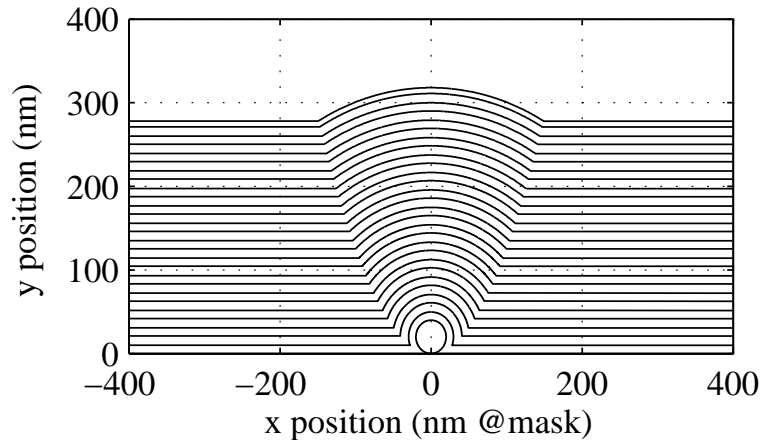

(a)

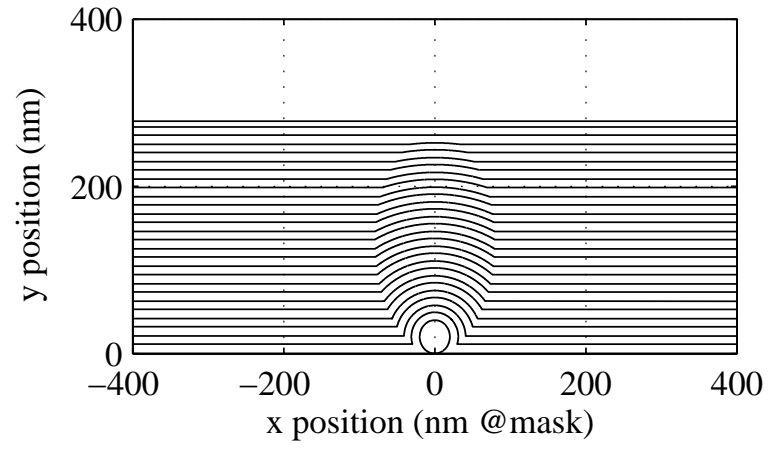

(b)

Figure 2

Patrick Schiavone, Renaud Payerne; "Rigorous simulation of line-defects in EUV masks" 


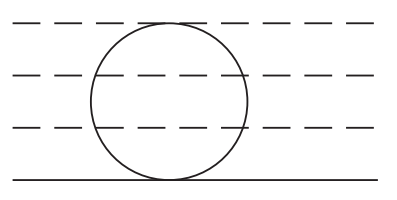

(a)

\begin{tabular}{c|c|c}
\hline $\mathrm{n} 1$ & $\mathrm{n} 2$ & $\mathrm{n} 1$ \\
\hline $\mathrm{n} 1$ & $\mathrm{n} 2$ & $\mathrm{n} 1$ \\
\hline $\mathrm{n} 1$ & $\mathrm{n} 2$ & $\mathrm{n} 1$ \\
\hline
\end{tabular}

(b)

Figure 3

Patrick Schiavone, Renaud Payerne; "Rigorous simulation of line-defects in EUV masks" 


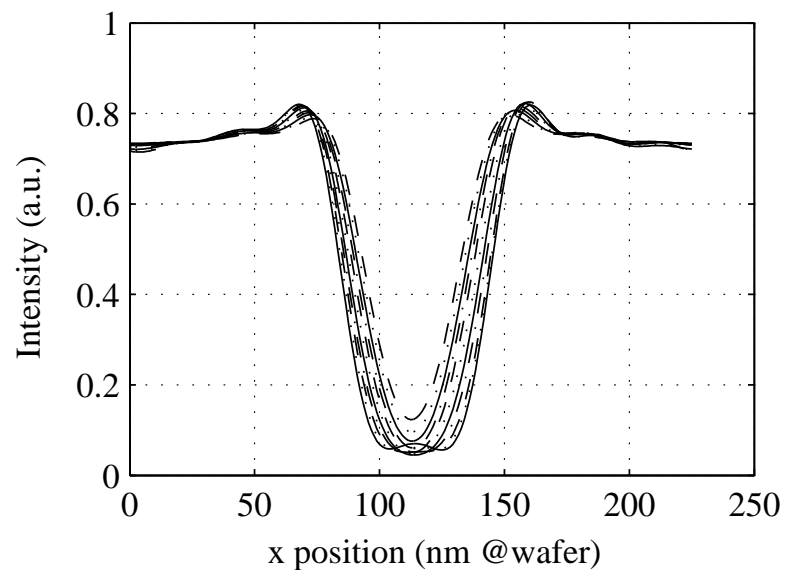

Figure 4

Patrick Schiavone, Renaud Payerne; "Rigorous simulation of line-defects in EUV masks" 


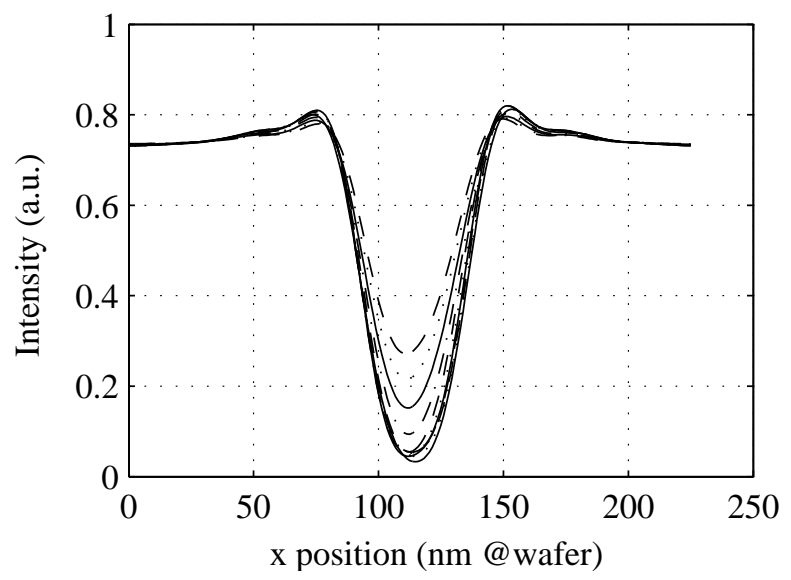

Figure 5

Patrick Schiavone, Renaud Payerne; "Rigorous simulation of line-defects in EUV masks" 


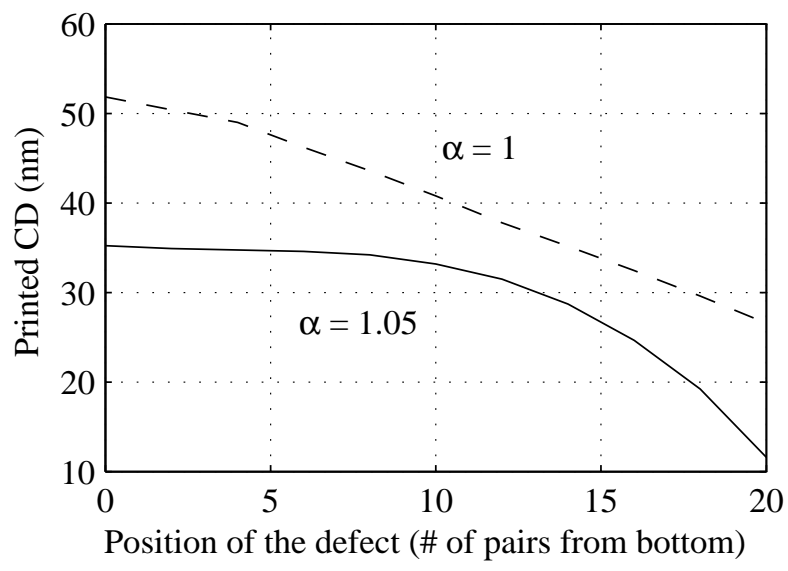

Figure 6

Patrick Schiavone, Renaud Payerne; "Rigorous simulation of line-defects in EUV masks" 


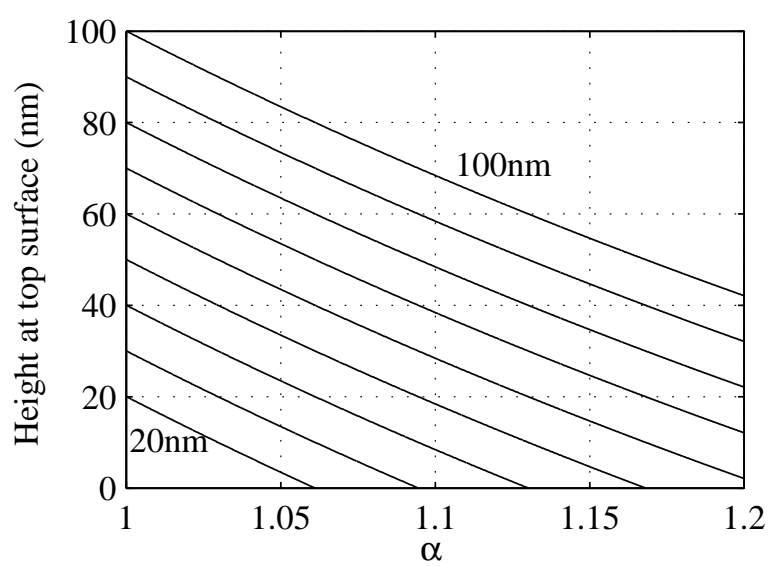

(a)

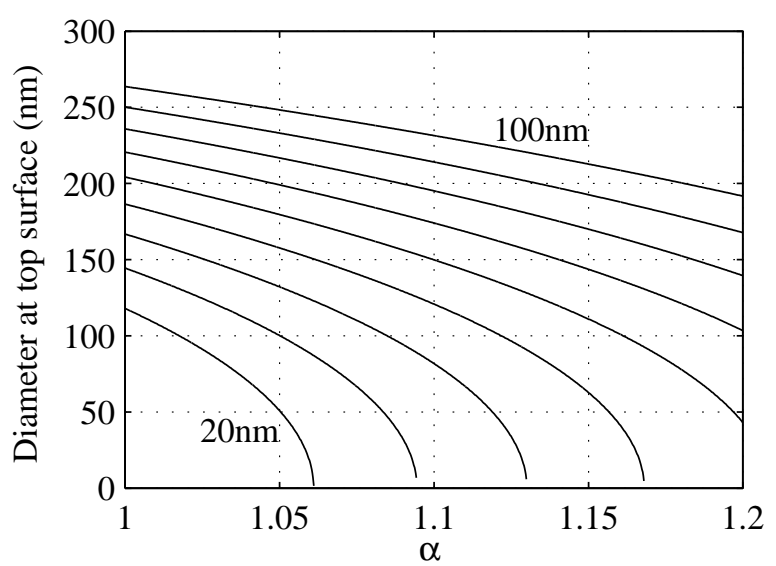

(b)

Figure 7

Patrick Schiavone, Renaud Payerne; "Rigorous simulation of line-defects in EUV masks" 


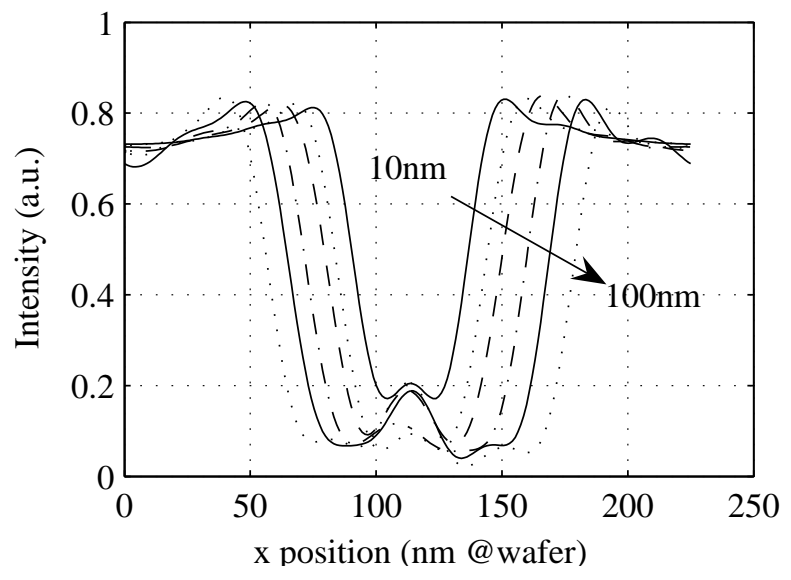

Figure 8

Patrick Schiavone, Renaud Payerne; "Rigorous simulation of line-defects in EUV masks" 


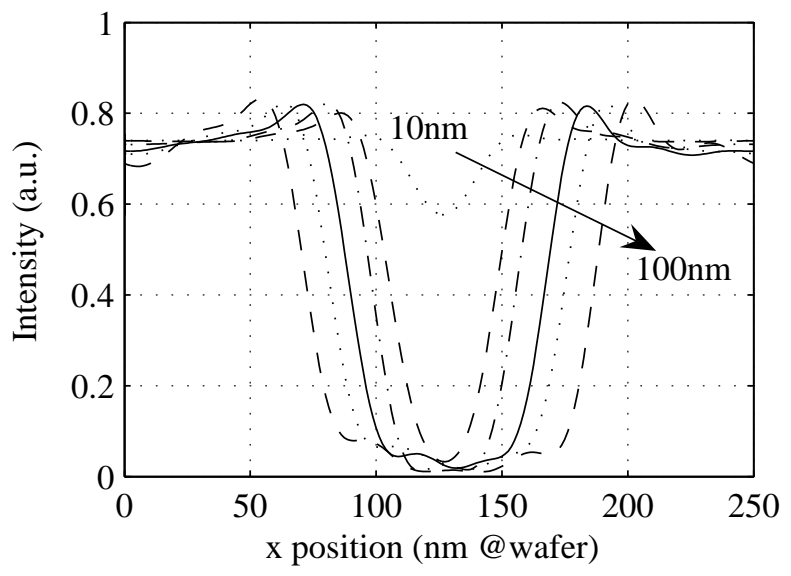

Figure 9

Patrick Schiavone, Renaud Payerne; "Rigorous simulation of line-defects in EUV masks" 


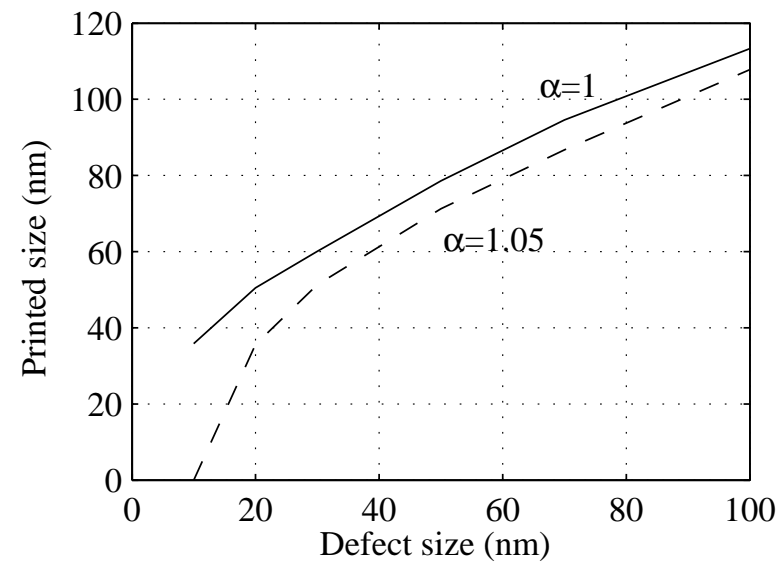

Figure 10

Patrick Schiavone, Renaud Payerne; "Rigorous simulation of line-defects in EUV masks" 


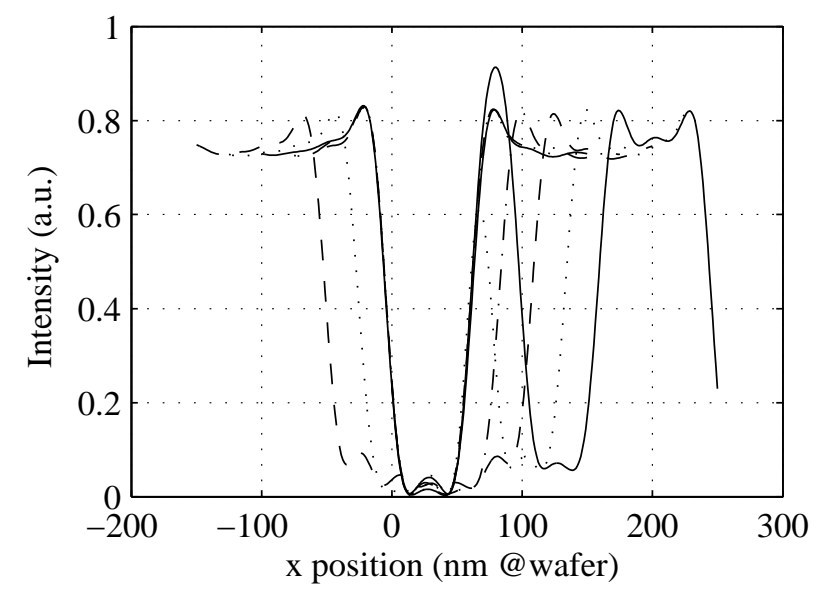

Figure 11

Patrick Schiavone, Renaud Payerne; "Rigorous simulation of line-defects in EUV masks" 


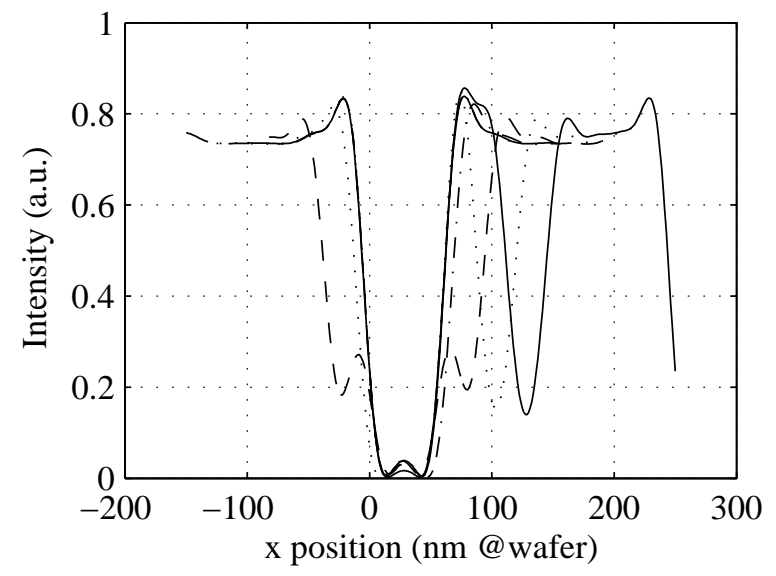

Figure 12

Patrick Schiavone, Renaud Payerne; "Rigorous simulation of line-defects in EUV masks" 


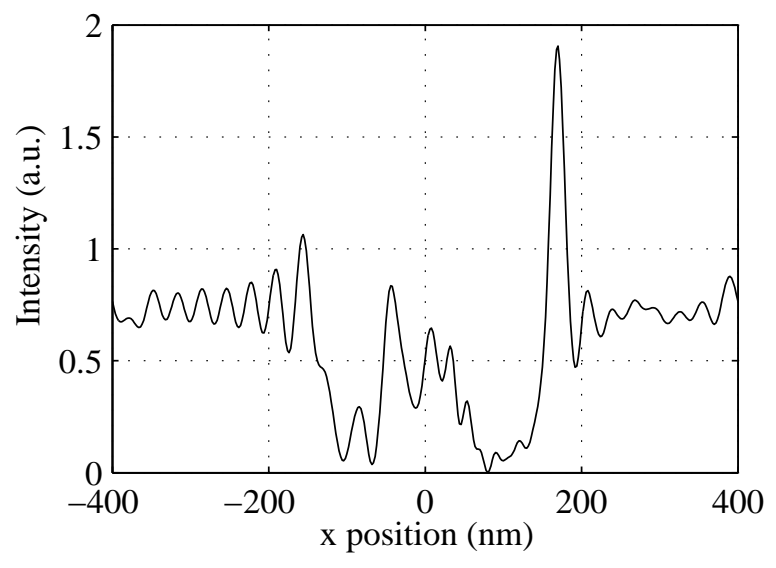

(a)

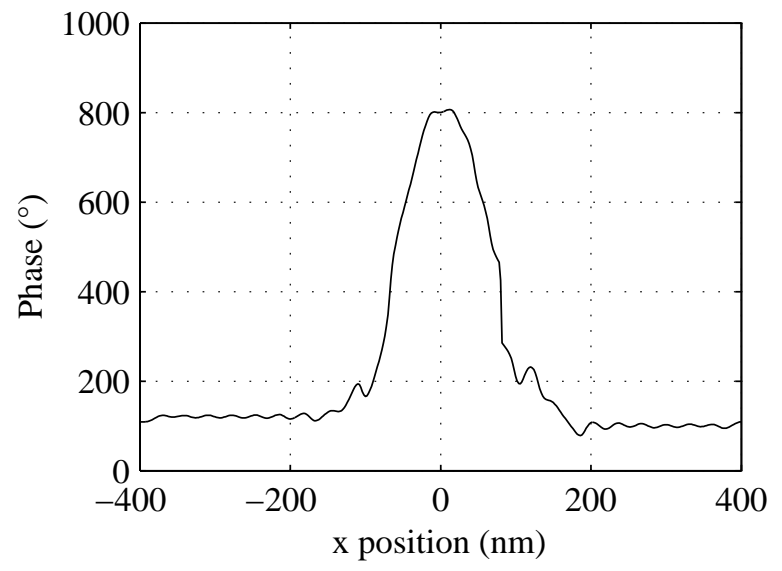

(b)

Figure 13

Patrick Schiavone, Renaud Payerne; "Rigorous simulation of line-defects in EUV masks" 


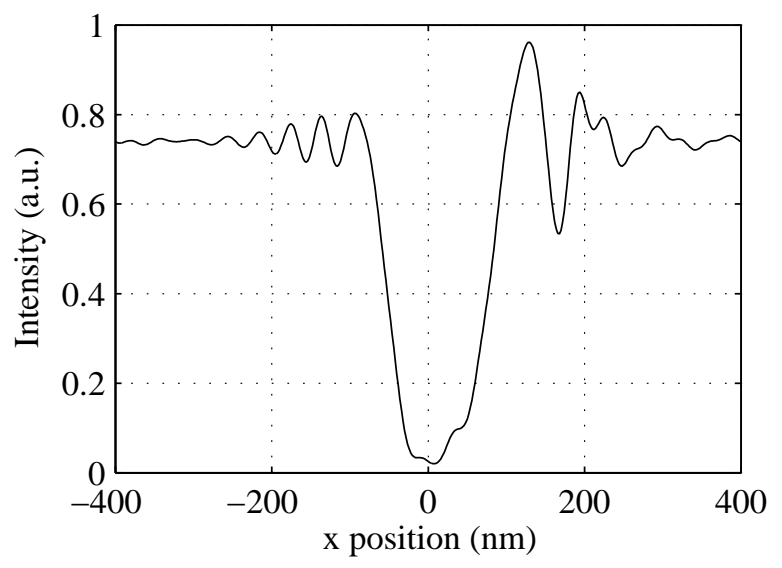

(a)

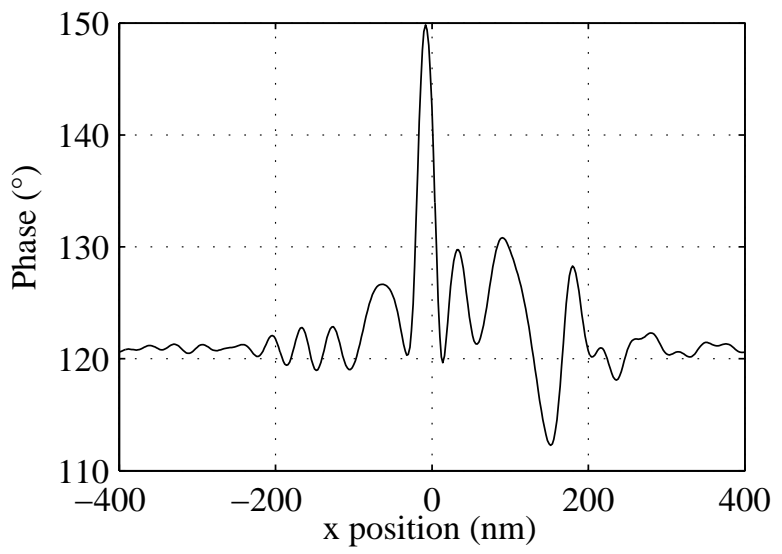

(b)

Figure 14

Patrick Schiavone, Renaud Payerne; "Rigorous simulation of line-defects in EUV masks" 


\section{Bibliography}

1) T. Pistor and A. Neureuther: J. Vac. Sci. Technol. B 17 (1999), 3019.

2) M. Ito, T. Ogawa, K. Otaki, W. Nishyama, S. Okazaki, and T. Terasawa: Jpn. J. Appl. Phys. 40 (2001), 2549.

3) E. M. Gullikson, C. Cerjan, D. G. Stearns, P. B. Mirkarimi, and D. W. Sweeney: J. Vac. Sci. Technol. B 20 (2002), 81.

4) P. Evanschitzky, A. Erdmann, M. Besacier, and P. Schiavone: Proc. SPIE Conf. Photomask and Next-Generation Lithography Mask Technology X, 2003, vol. 5130, p. 1035.

5) B. S. Bollepalli and F. Cerrina: Proc. SPIE Conf. Emerging Lithographic Technologies III, Santa Clara (USA), 1999, vol. 3676, p. 587.

6) X. Lee: http://www.xahlee.org/SpecialPlaneCurves_dir/Parallel_dir/parallel.html.

7) P. B. Mirkarimi, S. L. Baker, and D. G. Stearns: J. Vac. Sci. Technol. B 19 (2001)

8) P. Schiavone and G. Granet: Microelectronic Engineering 57-58 (2001), 497-503.

9) D. M. Pai and K. A. Awada: J. Opt. Soc. Am. A 8 (1991), 755.

10) K. Otaki: Jpn. J. Appl. Phys. 398 (2000), 6819.

11) P. B. Mirkarimi and D. G. Stearns: Appl. Phys. Lett. 77 (2000), 2243.

12) M. Yi, T. Haga, C. Walton, C. Larson, and J. Bokor: Jpn. J. Appl. Phys. 41 Part 1 (2002), 4101.

13) V. Farys, P. Schiavone, F. Polack, C. Vannuffel, E. Quesnel, and M. Bertolo: Proc. 2nd International EUV Lithography Symposium, Antwerp, 2003. 